\title{
Beauty Contests and Iterated Expectations in Asset Markets*
}

\author{
Franklin Allen \\ University of Pennsylvania \\ allenf@wharton . upenn . edu
}

\author{
Stephen Morris \\ Yale University \\ stephen.morris@yale.edu
}

Hyun Song Shin

London School of Economics

h.s.shin@lse.ac.uk

Revised, April 2004 
"...professional investment may be likened to those newspaper competitions in which the competitors have to pick out the six prettiest faces from a hundred photographs, the prize being awarded to the competitor whose choice most nearly corresponds to the average preferences of the competitors as a whole; so that each competitor has to pick, not those faces which he himself finds prettiest, but those which he thinks likeliest to catch the fancy of the other competitors, all of whom are looking at the problem from the same point of view. It is not a case of choosing those which, to the best of one's judgement, are really the prettiest, nor even those which average opinion genuinely thinks the prettiest. We have reached the third degree where we devote our intelligences to anticipating what average opinion expects the average opinion to be. And there are some, I believe, who practise the fourth, fifth and higher degrees." Keynes (1936), page 156. 


\section{Introduction}

Keynes (1936) introduced the influential metaphor of financial markets as a beauty contest. An implication of the metaphor is that an understanding of financial markets requires an understanding not just of market participants' beliefs about assets' future payoffs, but also an understanding of market participants' beliefs about other market participants' beliefs, and higher order beliefs. Judging by how often the above passage from Keynes is quoted in academic and non-academic circles, many people find the metaphor highly suggestive. Yet the theoretical literature on asset pricing has, until recently, failed to develop models that validate the role of higher order beliefs in asset pricing. The purpose of our paper is to illuminate the role of higher order expectations in an asset pricing context, and thereby to explore the extent to which Keynes's beauty contest metaphor is valid as a guide for thinking about asset prices.

Higher order expectations do not make an appearance in standard competitive asset pricing models with a representative investor. Asset prices in such settings reflect the discounted expected value of payoffs from the asset, suitably adjusted for risk. Since we believe that the beauty contest phenomenon alluded to above is consistent with competitive asset pricing models, our explanation must include an account of why asset prices in a competitive market may fail to reflect solely the discounted expected payoffs.

A key feature of the representative investor model of asset prices that makes higher order expectations redundant is the martingale property of asset prices. The price of an asset today is the discounted expected value of the asset's payoff stream with respect to an equivalent martingale measure, conditional on the information available to the representative individual today. This allows the folding back of future outcomes to the present in coming up with today's price. An implication of the martingale property in a representative individual economy is the law of iterated expectations in which 
the representative investor's expectation today of his expectation tomorrow of future payoffs is equal to his expectation today of future payoffs.

But if there is differential information between investors so that there is some role for the average expectations about payoffs, the folding back of future outcomes to the present cannot easily be achieved. In general, average expectations fail to satisfy the law of iterated expectations. It is not the case that the average expectation today of the average expectation tomorrow of future payoffs is equal to the average expectation of future payoffs. The key observation in this paper is not only that the law of iterated expectations fails to hold for average opinion when there is differential information, but that its failure follows a systematic pattern that gives rise to features of interest in finance.

Suppose that an individual has access to both private and public information about an asset's payoffs, and they are of equal value in predicting the asset's payoffs. In predicting the asset's payoffs, the individual would put equal weight on private and public signals. Now suppose that the individual is asked to guess what the average expectation of the asset's payoffs is. Since he knows that others have also observed the same public signal, the public signal is a better predictor of average opinion, he will put more weight on the public signal than on the private signal. Thus if individuals' willingness to pay for an asset is related to their expectations of average opinion, then we will tend to have asset prices overweighting public information relative to the private information. Thus any model where higher order beliefs play a role in pricing assets will deliver the conclusion that there is an excess reliance on public information.

This bias towards the public signal is reminiscent of the result in Morris and Shin (2002) where the coordination motive of the agents induces a disproportionate role for the public signal. Although there is no explicit coordination motive in the rational expectations equilibrium, the fact that the public signal enters into everyone's demand function means that it still 
retains some value for forecasting the aggregate demand above and beyond its role in estimating the liquidation value. Another way of expressing this is to say that, whereas the noise in the individual traders' private signals get "washed out" when demand is aggregated across traders, the noise term in the public signal is not similarly washed out. Thus, the noise in the public signal is still useful in forecasting aggregate demand, and hence the price.

Grossman (1976), Hellwig (1980) and Diamond and Verrecchia (1981) showed how equilibrium can be constructed when prices play an informational role in competitive asset markets with differential information. Admati (1985) extended the analysis to a multi-asset setting and showed the importance of correlations between the traders' prediction errors in determining asset returns, over and above the traditional emphasis on the correlation of asset returns.

Our main focus, however, is on the dynamic, multi-period asset pricing context with a single risky asset. Do asset prices reflect average opinion, and average opinion about average opinion, in the manner that Keynes suggests? Our answer is a resounding "yes", and we construct a standard asset pricing model where this is the case. We look at a version of the dynamic, noisy rational expectations asset pricing model, of the type developed by Singleton (1987), Brown and Jennings (1989), Grundy and McNichols (1989) and He and Wang $(1995)^{1}$. Much of the early literature has focussed on trading volume and has not highlighted the role of higher order beliefs in asset prices Our purpose is to show how higher order beliefs are reflected in asset prices ${ }^{2}$.

\footnotetext{
${ }^{1}$ Wang (1993) examines a model where some traders are strictly better informed than the rest, so that the informed traders' information is a sufficient statistic for the economy.

${ }^{2}$ Although the early literature did not address higher order beliefs explicitly, Brown and Jennings (1989) did focus on asset pricing issues, showing that in noisy rational expectations equilibria (unlike in a martingale asset pricing world), past prices convey information to market participants. He and Wang (1995) note (in the discussion following their Lemma 3) how their finite horizon assumption allows higher order expectations to be reduced to lower order expectations in solving the model. Our purpose in this paper is to show how and why equilibrium price relationships in dynamic noisy rational expectations models, such as those of Brown and Jennings (1989) and He and Wang (1995), reflect the
} 
In our model, there is a single risky asset that will be liquidated at date $T+1$, but is traded at dates 1 to $T$ by overlapping generations of traders who trade when young, and consume when old. Our assumption of overlapping generations of traders is intended to accentuate the importance of short-run price movements for traders. Even for long-lived traders, if they have a preference for smoothing consumption over time, they will care about short-run price movements, as well as the underlying fundamental value of the asset at its ultimate liquidation. For example, one reason for short horizons is that individuals' funds are managed by professionals and inefficiencies resulting from the agency problem give rise to short horizons (Allen and Gorton (1993)). In the concluding section (section 4), we discuss some of the existing literature with similar conclusions. The assumption of overlapping generations is used as a device to throw into sharper relief this concern for short-run prices. Our motivation for making this assumption is to attempt to capture some of the intuition behind Keynes's beauty contests metaphor, in which traders are motivated to second and third-guess other traders in order to profit from short-run price movements. Our assumption of overlapping generations of traders makes it similar to the short-lived trader version of the model examined in Brown and Jennings (1989).

By developing a framework of analysis that can accommodate higher order beliefs, we show how Keynes's beauty contest metaphor can be formalized in our setting, and how it has a direct impact on asset prices. There are two themes to our results.

- First, the mean path of prices will, in general, depart from the market consensus of the expected fundamental value of the asset. Even though prices result from forward-looking expectations, there is no unravelling of the iterated expectations to force prices to be the same as consensus expectations of fundamentals. To the extent that the mean price path higher order beliefs intuition of Keynes' beauty contest metaphor. 
deviates from the consensus liquidation value, we have elements of a bubble.

- Second, prices in a beauty contest react much more sluggishly to changes in the fundamental value of the asset. Prices exhibit inertia, or "drift". In particular, prices react more sluggishly to changes in fundamentals than does the consensus fundamental value. Even as traders update their views of the fundamental value, the price does not react as much.

The second bullet point is reminiscent of the literature on the apparent underreaction of asset prices over short horizons. An enduring empirical regularity that has received much attention is that, over horizons of perhaps one to twelve months, there is a predictable component to asset returns where asset prices exhibit momentum ${ }^{3}$. In a beauty contest, prices exhibit the same outward sign of the momentum or drift. Thus, even though prices are forward-looking expectations of future liquidation, we find considerable inertia in such forward-looking expectations.

The insights of this paper are relevant beyond the asset pricing application. The macroeconomics literature on the forecasting the forecasts of others as initiated by Townsend $(1978,1983)$ and Phelps $(1983)$, and developed by Sargent (1991) and others, looked at dynamic models where agents follow linear decision rules but their choices depend on others' choices and their heterogeneous expectations about future realizations of economic variables. As a consequence, forward-looking iterated average expectations matter. The CARA-normal noisy rational expectations asset prices of this paper inherit both the linear decision rules and the forward looking iterated average expectations. One insight highlighted in this paper is that forward looking iterated average expectations exhibit inertia, and is relevant for the recently renewed interest in heterogeneous expectations in macroeconomics where this insight

\footnotetext{
${ }^{3}$ There is a very large empirical literature. Barberis, Shleifer and Vishny (1998) gathers together some of the evidence.
} 
might be relevant ${ }^{4}$. Explicit solutions for such macroeconomic models are rarely possible due to their complexity. In contrast, the questions addressed in our paper are sufficiently simple for us to derive a number of explicit results. These results may have some bearing on the general problem of the role of iterated expectations in differential information economies.

Before launching into our asset pricing model, we provide some background by examining the abstract properties of higher order expectations in section 2. Our main asset pricing analysis follows this initial discussion. We conclude in section 4 by discussing the robustness of our model to changes in assumptions (in particular, relaxing the short-lived trader assumption), and relating our results to some of the recent literature.

\section{Background}

For any random variable $\theta$, let $E_{i t}(\theta)$ be player $i$ 's expectation of $\theta$ at date $t$; write $\bar{E}_{t}(\theta)$ for the average expectation of $\theta$ at time $t$; and write $E_{t}^{*}(\theta)$ for the public expectation of $\theta$ at time $t$ (i.e., the expectation of $\theta$ conditional on public information only; in a partition model, this would be conditional on the meet of players' information).

We know that individual and public expectations satisfy the law of iterated expectations:

$$
\begin{aligned}
E_{i t}\left(E_{i, t+1}(\theta)\right) & =E_{i t}(\theta) \\
\text { and } E_{t}^{*}\left(E_{t+1}^{*}(\theta)\right) & =E_{t}^{*}(\theta) .
\end{aligned}
$$

But the analogous property for average expectations will typically fail under asymmetric information. In other words, we will typically have

$$
\bar{E}_{t}\left(\bar{E}_{t+1}(\theta)\right) \neq \bar{E}_{t}(\theta) .
$$

\footnotetext{
${ }^{4}$ For example, Amato and Shin (2003), Hellwig (2002), Stasavage (2002) and Woodford (2003). Pearlman and Sargent (2002) show that some cases of such problems reduce to the common knowledge case.
} 
This is most easily seen by considering the case where there is no learning over time. Suppose $\theta$ is distributed normally with mean $y$ and variance $\frac{1}{\alpha}$. Each player $i$ in a continuum observes a signal $x_{i}=\theta+\varepsilon_{i}$, where $\varepsilon_{i}$ is distributed in the population with mean 0 and variance $\frac{1}{\beta}$. Suppose that this is all the information available at all dates. Then we may drop the date subscripts. Now observe that

$$
\begin{aligned}
E_{i}(\theta) & =\frac{\alpha y+\beta x_{i}}{\alpha+\beta} \\
\bar{E}(\theta) & =\frac{\alpha y+\beta \theta}{\alpha+\beta} \\
E_{i}(\bar{E}(\theta)) & =\frac{\alpha y+\beta E_{i}(\theta)}{\alpha+\beta} \\
& =\frac{\alpha y+\beta\left(\frac{\alpha y+\beta x_{i}}{\alpha+\beta}\right)}{\alpha+\beta} \\
& =\left(1-\left(\frac{\beta}{\alpha+\beta}\right)^{2}\right) y+\left(\frac{\beta}{\alpha+\beta}\right)^{2} x_{i} \\
\bar{E}(\bar{E}(\theta)) & =\left(1-\left(\frac{\beta}{\alpha+\beta}\right)^{2}\right) y+\left(\frac{\beta}{\alpha+\beta}\right)^{2} \theta
\end{aligned}
$$

Iterating this operation, one can show that

$$
\bar{E}^{k}(\theta)=\left(1-\left(\frac{\beta}{\alpha+\beta}\right)^{k}\right) y+\left(\frac{\beta}{\alpha+\beta}\right)^{k} \theta
$$

Note that (1) the expectation of the expectation is biased towards the public signal $y$ : that is,

$$
\operatorname{sign}(\bar{E}(\bar{E}(\theta))-\bar{E}(\theta))=\operatorname{sign}(y-\bar{E}(\theta))
$$

and (2) as $k \rightarrow \infty, \bar{E}^{k}(\theta) \rightarrow y \cdot{ }^{5}$ Putting back the time subscripts, we have

$$
\bar{E}_{t}\left(\bar{E}_{t+1}(\theta)\right)=\left(1-\left(\frac{\beta}{\alpha+\beta}\right)^{2}\right) y+\left(\frac{\beta}{\alpha+\beta}\right)^{2} \theta \neq \frac{\alpha y+\beta \theta}{\alpha+\beta}=\bar{E}_{t}(\theta) .
$$

${ }^{5}$ Property (1) does not hold for all distributions: one can construct examples where it fails to hold. However, property (2) holds independently of the normality assumption: this is, for any random variable and information system with a common prior, the average expectation of the average expectations.... of the random variable converges to the expectation of the random variable conditional on public information (see Samet (1998)). 
and

$$
\bar{E}_{t}\left(\bar{E}_{t+1}\left(\ldots \ldots \bar{E}_{T-2}\left(\bar{E}_{T-1}(\theta)\right)\right)\right)=\left(1-\left(\frac{\beta}{\alpha+\beta}\right)^{T-t}\right) y+\left(\frac{\beta}{\alpha+\beta}\right)^{T-t} \theta .
$$

Now suppose that there is an asset that has liquidation value $\theta$ at date $T$. Suppose - in the spirit of the Keynes beauty contest - that the asset is priced according to the asset pricing formula

$$
p_{t}=\bar{E}_{t}\left(p_{t+1}\right) \text {. }
$$

Then we would have

$$
p_{t}=\left(1-\left(\frac{\beta}{\alpha+\beta}\right)^{T-t}\right) y+\left(\frac{\beta}{\alpha+\beta}\right)^{T-t} \theta \text {. }
$$

Given the realization of the public signal, the period $t$ price is biased toward the public signal relative to fundamentals. It also implies that, unconditional on the realized public signal, the period $t$ price is normally distributed with mean $\theta$ and variance $\frac{1}{\alpha}\left(1-\left(\frac{\beta}{\alpha+\beta}\right)^{T-t}\right)$. Thus the more trading periods there are, the higher the variance of the price. This is despite the fact that (by assumption) no new information is being revealed.

There is an alternative way to present the argument that is revealing, and which also anticipates our general argument later. Let us recast the inference problem for individual $i$ in matrix form as follows.

$$
E_{i}\left[\begin{array}{l}
y \\
\theta
\end{array}\right]=\left[\begin{array}{cc}
1 & 0 \\
\frac{\alpha}{\alpha+\beta} & \frac{\beta}{\alpha+\beta}
\end{array}\right]\left[\begin{array}{c}
y \\
x_{i}
\end{array}\right]
$$

Averaging over individuals,

$$
\bar{E}\left[\begin{array}{l}
y \\
\theta
\end{array}\right]=\left[\begin{array}{cc}
1 & 0 \\
\frac{\alpha}{\alpha+\beta} & \frac{\beta}{\alpha+\beta}
\end{array}\right]\left[\begin{array}{l}
y \\
\theta
\end{array}\right]
$$

Thus, the averge belief operator $\bar{E}$ can be represented by the transition matrix of a two state Markov chain defined over the state space $\{y, \theta\}$. Note that the public signal $y$ is an absorbing state in this Markov chain, since once 
the system has entered the state, it never leaves. Then, higher order average expectations can be obtained by iteration of the transition matrix.

$$
\begin{aligned}
\bar{E}^{k}\left[\begin{array}{l}
y \\
\theta
\end{array}\right] & =\left[\begin{array}{cc}
1 & 0 \\
\frac{\alpha}{\alpha+\beta} & \frac{\beta}{\alpha+\beta}
\end{array}\right]^{k}\left[\begin{array}{l}
y \\
\theta
\end{array}\right] \\
& =\left[\begin{array}{cc}
1 & 0 \\
1-\left(\frac{\beta}{\alpha+\beta}\right)^{k} & \left(\frac{\beta}{\alpha+\beta}\right)^{k}
\end{array}\right]\left[\begin{array}{l}
y \\
\theta
\end{array}\right] \\
& \rightarrow\left[\begin{array}{l}
y \\
y
\end{array}\right] \quad \text { as } k \rightarrow \infty
\end{aligned}
$$

The weight on the true value of $\theta$ is the probability of being in a transitory state in the Markov chain. Hence, as the order of beliefs increases, the true value of $\theta$ receives less and less weight.

We will now show how this Markov chain insight can be used in the main analysis. So far, we have given no justification for asset pricing formula (2), other than an appeal to the authority of Keynes. We would like to describe an asset pricing model that generates asset pricing formula (2), or something like it, and deals with the issue of learning from prices. We will turn to this problem now.

\section{Asset Prices}

Time is discrete, and is labelled by the set $\{1,2, \cdots, T, T+1\}$. There is a single risky asset that will be liquidated at date $T+1$, but is traded at dates 1 to $T$. The liquidation value $\theta$ of the asset is determined before trading at date 1 , and is a normally distributed random variable with mean $y$ and variance $1 / \alpha$. Once $\theta$ is determined at date 1 , it remains fixed until the asset is liquidated at date $T+1$. There are overlapping generations of traders who each live for two periods. A new generation of traders of unit measure is born at each date $t$, and are indexed by the unit interval $[0,1]$. At date $t$, when they are young, they trade the asset to build up a position in the asset, but do not consume. We assume that they have sufficient endowments so that 
they are never wealth constrained. In the next period (at date $t+1$ ) when they are old, they unwind their asset holding and acquire the consumption good, consume and die. Thus, at any trading date $t$, there is a unit mass of young traders and a unit mass of old traders.

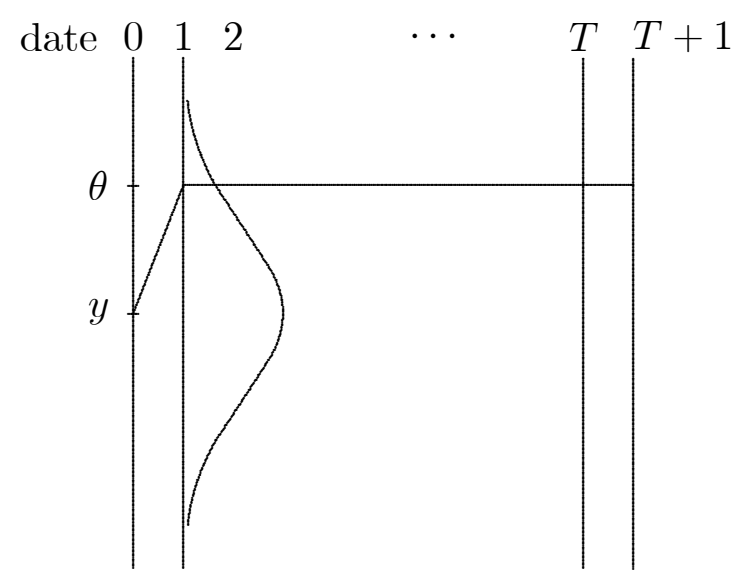

Figure 1: Path of fundamental value

Our assumption of overlapping generations of traders is intended to accentuate the importance of short-run price movements for traders. Even for long-lived traders, if they have a preference for smoothing consumption over time, they will care about short-run price movements, as well as the underlying fundamental value of the asset at its ultimate liquidation. The assumption of overlapping generations is used as a device to throw into sharper relief this concern for short-run prices. Our motivation for making this assumption is to attempt to capture some of the intuition behind Keynes's beauty contests metaphor, in which traders are motivated to second and third-guess other traders in order to profit from short-run price movements. Our assumption of overlapping generations of traders makes it similar to the short-lived trader version of the model examined in Brown and Jennings $(1989)^{6}$.

\footnotetext{
${ }^{6}$ In contrast, other authors such as He and Wang (1995) have examined the case where traders only consume at the terminal date, and trade up to that date.
} 


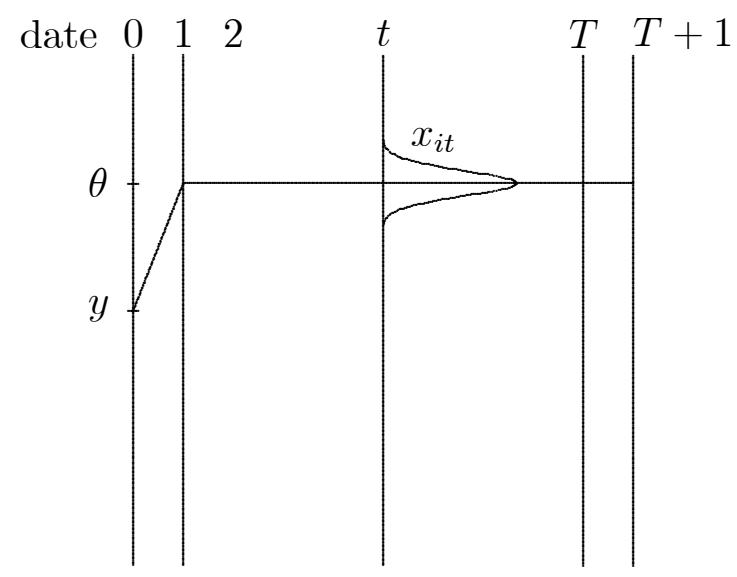

Figure 2: Private information

When each new trader is born, they do not know the true value of $\theta$. However, for a trader $i$ born at date $t$, there are two sources of information. First, the full history of past and current prices are available, including the ex ante mean $y$ of $\theta$. Second, this trader observes the realization of a private signal

$$
x_{i t}=\theta+\varepsilon_{i t}
$$

where $\varepsilon_{i t}$ is a normally distributed noise term with mean 0 and variance $1 / \beta$. We assume that the noise terms $\left\{\varepsilon_{i t}\right\}$ are i.i.d. across individuals $i$ and across time $t$. There is no other source of information for the trader. In particular, the private signals of the previous generation of traders are not observable. Hence, the information set of trader $i$ at date $t$ is

$$
\left\{y, p_{1}, p_{2}, \cdots, p_{t}, x_{i t}\right\}
$$

where $p_{t}$ is the price of the asset at date $t$. As a convention, we take $p_{T+1}=\theta$. All traders have the exponential utility function $u(c)=-e^{-\frac{c}{\tau}}$ defined on consumption $c$ when they are old. The parameter $\tau$ is the reciprocal of the absolute risk aversion, and we shall refer to it as the traders' risk tolerance.

Finally, in each trading period, we assume that there is an exogenous 
noisy supply of the asset, $s_{t}$, distributed normally with mean 0 and precision $\gamma_{t}$. The supply noise is independent over time, and independent of the fundamentals and the noise in traders' information.

The role of iterated expectations can be brought out in our context. Begin with a trader $i$ who trades at the final trading date $T$. The trader's demand for the asset is linear due to constant absolute risk aversion and normally distributed payoffs and is given by

$$
\frac{\tau}{\operatorname{Var}_{i T}(\theta)}\left(E_{i T}(\theta)-p_{T}\right)
$$

where $E_{i T}(\theta)$ is the expectation of $\theta$ conditional on trader $i$ 's information set at date $T$, and $\operatorname{Var}_{i T}(\theta)$ is the conditional variance of $\theta$ with respect to the same information set. Because the traders have private signals that are i.i.d. conditional on $\theta$, the conditional variances $\left\{\operatorname{Var}_{i T}(\theta)\right\}$ are identical across traders, and we denote by $\operatorname{Var}_{T}(\theta)$ this common conditional variance across traders. Summing (4) across traders, the aggregate demand at date $T$ is given by

$$
\frac{\tau}{\operatorname{Var}_{T}(\theta)}\left(\bar{E}_{T}(\theta)-p_{T}\right)
$$

where $\bar{E}_{T}(\theta)$ is the average expectation of $\theta$ at date $T$. Market clearing then implies that

$$
p_{T}=\bar{E}_{T}(\theta)-\frac{\operatorname{Var}_{T}(\theta)}{\tau} s_{T}
$$

The price at the previous date $T-1$ can be derived from an analogous argument, bearing in mind that young traders who trade at date $T-1$ care about $p_{T}$ rather than the final liquidation value of the asset, since their own consumption is related to $p_{T}$. The price at date $T-1$ is given by

$$
p_{T-1}=\bar{E}_{T-1}\left(p_{T}\right)-\frac{\operatorname{Var}_{T-1}\left(p_{T}\right)}{\tau} s_{T-1}
$$

Substituting (6) into (7), and noting that $\bar{E}_{T-1}\left(s_{T}\right)=0$, we have the following expression for price at $T-1$.

$$
p_{T-1}=\bar{E}_{T-1} \bar{E}_{T}(\theta)-\frac{\operatorname{Var}_{T-1}\left(p_{T}\right)}{\tau} s_{T-1}
$$


This is a recursive relationship that can be iterated further back in time. Thus, the price at date $t$ is given by

$$
p_{t}=\bar{E}_{t} \bar{E}_{t+1} \cdots \bar{E}_{T}(\theta)-\frac{\operatorname{Var}_{t}\left(p_{t+1}\right)}{\tau} s_{t}
$$

The price at date $t$ is the average expectation at date $t$ of the average expectation at the next trading date, etc. of the final liquidation value $\theta$. Expressions such as (9) should encourage us that we have an approach that may enable us to address Keynes's beauty contest metaphor. However, we need to delve deeper into the consequences of the iterated expectations in order to make any headway. In particular, we would like to address two questions.

- Is there any systematic difference between the price $p_{t}$ and the consensus value of the fundamentals given by $\bar{E}_{t}(\theta)$ ? The term "systematic" is important here, since the noisy supply will mean that the actual realization of the price is noisy. Rather, the question is whether, averaging over the noisy realizations of the supply, the price deviates systematically from the average expectations of the fundamentals. If so, we have elements of a bubble to the extent that the market price does not reflect the consensus about the true value of the fundamentals.

- How quickly does the price adjust to the shift in fundamentals at date 1 ? Does the price adjust rapidly to take account of the new fundamental value, or is there inertia or "drift" in the path followed by prices? In particular, how does the speed of the adjustment of the price path differ from the speed of the adjustment of the average expectations of fundamentals?

We will answer these questions by means of three propositions. Figure 3 illustrates our results. The line labelled as $p_{t}$ is the mean of the price path with respect to the noisy realizations of supply. The line labelled as 


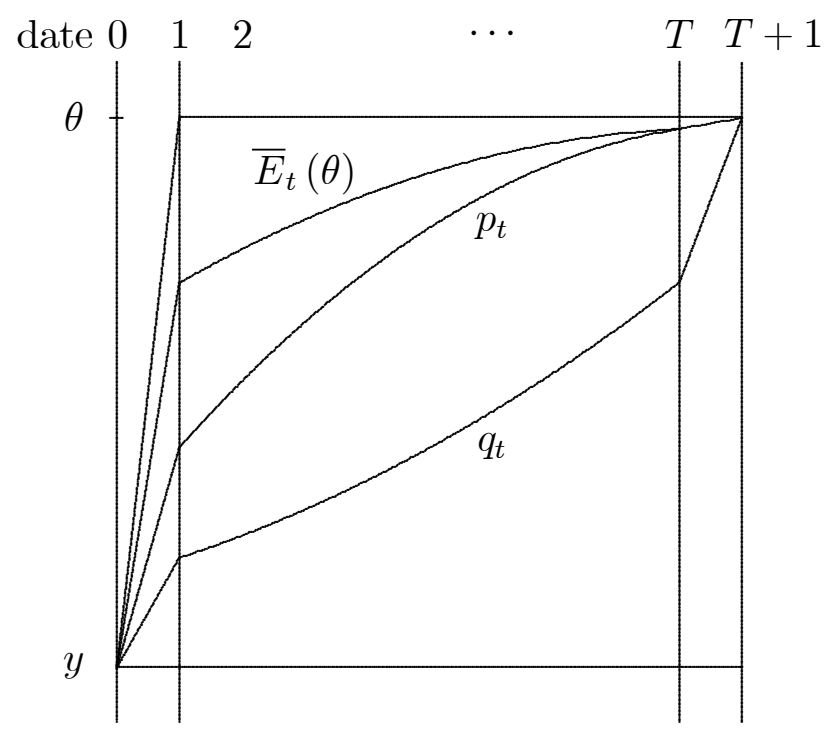

Figure 3: Mean of time paths of $p_{t}$ and $\bar{E}_{t}(\theta)$

$\bar{E}_{t}(\theta)$ is the mean path of the average expectation of $\theta$ with respect to the noisy realizations of supply. The mean price path deviates systematically from the mean path of average expectations, and the price path lies further away from the true value than the average expectations. So, prices not only fail to reflect the consensus on true fundamental value, but is further away from the true value. Also, the initial adjustment in price at date 1 is too sluggish, failing to reflect the true extent of the shift in fundamentals from $y$ to $\theta$. Thereafter, the price does adjust, but slowly. It only catches up with the average expectations of fundamentals at the last trading date. The line labelled by $q_{t}$ is the path followed by naive iterated expectation given by equation (3) in section 2 that ignores the information given by prices. The mean path for price lies between this path and the mean path for average expectations. One of our results below identifies a limiting case for when the mean price path coincides with the naive iterated expectations path.

In what follows, we denote by $E_{s}(\cdot)$ the expectations with respect to the noisy supply terms $\left\{s_{t}\right\}$. The propositions below are proved in appendix A. 
Proposition 1 For all $t<T$

$$
E_{s}\left(\left|p_{t}-\theta\right|\right)>E_{s}\left(\left|\bar{E}_{t}(\theta)-\theta\right|\right)
$$

It is only at the final trading date $T$, that we have $E_{s}\left(p_{T}\right)=E_{s}\left(\bar{E}_{T}(\theta)\right)$.

Prices deviate systematically from the average expectations of the fundamental value of the asset. Even though price is a forward-looking expectation of the final liquidation value, the beauty contests element of the problem prevents the folding back of the final liquidation value into prices. The proof of proposition 1 in appendix A reveals that the root cause of the deviation of price from the average expectation of the fundamental value can be traced to the the excess weight given to past prices and the ex ante mean of $\theta$ in the determination of price, where "excess" is defined relative to the statistically optimal forecast of the fundamental value $\theta$.

Proposition 2 There exist weights $\left\{\lambda_{t}\right\}$ with $0<\lambda_{T}<\lambda_{T-1}<\cdots<\lambda_{2}<$ $\lambda_{1}<1$ such that

$$
E_{s}\left(p_{t}\right)=\lambda_{t} y+\left(1-\lambda_{t}\right) \theta
$$

Prices exhibit inertia, of "drift" in adjusting to the fundamental value $\theta$. Taken together with proposition 1 , this implies that after fundamentals are realized at date 1 , the price does not adjust sufficiently. Instead, it approaches the true fundamental value slowly in incremental steps. Such a price path would have many outward appearances of short run momentum in prices, as documented in many empirical studies.

Proposition 3 Let $q_{t}$ be defined as

$$
q_{t}=\left(1-\left(\frac{\beta}{\alpha+\beta}\right)^{T-t+1}\right) y+\left(\frac{\beta}{\alpha+\beta}\right)^{T-t+1} \theta
$$

Then, as $\tau \rightarrow 0$, we have

$$
E_{s}\left(p_{t}\right) \rightarrow q_{t}
$$


The sequence $\left\{q_{t}\right\}$ is the naive iterated exectations that we encountered in equation (3) in section 2. As traders become more and more risk averse, the mean price path approaches the naive iterated expectations where prices do not figure at all in the inference about the fundamentals. As we will see below, the intuition for the result lies in the fact as traders become more risk averse, they become less aggressive in setting their demand functions, thereby making prices less informative. In the limit, prices cease to have any information value at all.

We will argue for our results by presenting a framework that uses the intuitions from the Markov chain argument that we saw in section 2. We begin with the expression for $p_{t}$ in terms of the iterated average expectations.

$$
p_{t}=\bar{E}_{t} \bar{E}_{t+1} \cdots \bar{E}_{T}(\theta)-\frac{\operatorname{Var}_{t}\left(p_{t+1}\right)}{\tau} s_{t}
$$

From joint normality of the random variables, $E_{i t} \bar{E}_{t+1} \cdots \bar{E}_{T}(\theta)$ is a linear combination $l\left(x_{i}, y, p_{1}, p_{2}, \cdots, p_{t}\right)$ of random variables in $i$ 's information set. Taking averages across $i, \bar{E}_{t} \bar{E}_{t+1} \cdots \bar{E}_{T}(\theta)$ is given by $l\left(\theta, y, p_{1}, p_{2}, \cdots, p_{t}\right)$ the same linear combination in which $x_{i}$ is replaced by $\theta$. Thus, $p_{t}$ can be written as a linear function $L_{t}(\cdot)$ of $y, \theta, s_{t}$ and past prices.

$$
\begin{aligned}
p_{1}= & L_{1}\left(y, \theta, s_{1}\right) \\
p_{2}= & L_{2}\left(y, \theta, s_{2}, p_{1}\right) \\
& \vdots \\
p_{T}= & L_{T}\left(y, \theta, s_{T}, p_{1}, p_{2}, \cdots, p_{T}\right)
\end{aligned}
$$

Then $p_{2}=L_{2}\left(y, \theta, s_{2}, L_{1}\left(y, \theta, s_{1}\right)\right)$, which is a linear function of $y, \theta, s_{1}$ and $s_{2}$. By recursive substitution, $p_{t}$ can be expressed as a linear combination of $\left\{y, \theta, s_{1}, s_{2}, \cdots, s_{t}\right\}$. Thus,

$$
\begin{aligned}
& p_{1}=\lambda_{1} y+\kappa_{1} \theta-\phi_{11} s_{1} \\
& p_{2}=\lambda_{2} y+\kappa_{2} \theta-\phi_{21} s_{1}-\phi_{22} s_{2}
\end{aligned}
$$




$$
p_{T}=\lambda_{T} y+\kappa_{T} \theta-\phi_{T 1} s_{1}-\phi_{T 2} s_{2}-\cdots-\phi_{T T} s_{T}
$$

Let us write (10) in matrix form as follows.

$$
\left[\begin{array}{c}
p_{1} \\
\vdots \\
p_{T}
\end{array}\right]=\left[\begin{array}{c}
\lambda_{1} \\
\vdots \\
\lambda_{T}
\end{array}\right] y+\left[\begin{array}{c}
\kappa_{1} \\
\vdots \\
\kappa_{T}
\end{array}\right] \theta-\left[\begin{array}{ccc}
\phi_{11} & 0 & 0 \\
\vdots & \ddots & 0 \\
\phi_{T 1} & \cdots & \phi_{T T}
\end{array}\right]\left[\begin{array}{c}
s_{1} \\
\vdots \\
s_{T}
\end{array}\right]
$$

The matrix $\Phi$ of coefficients $\left\{\phi_{s t}\right\}$ is lower triangular, and hence invertible. Multiplying both sides by the inverse and re-arranging, we can obtain random variables $\left\{\xi_{1}, \xi_{2}, \cdots, \xi_{T}\right\}$ that are independent conditional on $\theta$.

$$
\begin{aligned}
\xi_{1} \equiv & \frac{\phi_{11}^{(-1)}\left(p_{1}-\lambda_{1} y\right)}{\phi_{11}^{(-1)} \kappa_{1}}=\theta-\frac{s_{1}}{\phi_{11}^{(-1)} \kappa_{1}} \\
& \vdots \\
\xi_{T} \equiv & \frac{\phi_{T 1}^{(-1)}\left(p_{1}-\lambda_{1} y\right)+\cdots+\phi_{T T}^{(-1)}\left(p_{T}-\lambda_{T} y\right)}{\phi_{T 1}^{(-1)} \kappa_{1}+\cdots+\phi_{T T}^{(-1)} \kappa_{T}}=\theta-\frac{s_{T}}{\phi_{T 1}^{(-1)} \kappa_{1}+\cdots+\phi_{T T}^{(-1)} \kappa_{T}}
\end{aligned}
$$

where $\phi_{s t}^{(-1)}$ denotes the $(s, t)$ th entry of $\Phi^{-1}$. Here, $\xi_{t}$ can be seen as a random variable that is a noisy signal of $\theta$ conveyed by price $p_{t}$. $\xi_{t}$ is normally distributed with mean $\theta$, and some given precision $\rho_{t}$. The precision $\rho_{t}$ of this signal is, of course, endogenously determined in equilibrium, and a full solution of the rational expectations equilibrium will entail solving for the precisions of all signals $\xi_{t}$. However, for our qualitative results below, a full solution turns out not to be necessary.

By using the transformed variables, trader $i$ 's information set at date $t$ can be written as $\left\{x_{i}, y, \xi_{1}, \xi_{2}, \cdots, \xi_{t}\right\}$. The expectation of $\theta$ conditional on this information set is given by

$$
E_{i t}(\theta)=\frac{\beta x_{i}+\alpha y+\sum_{i=1}^{t} \rho_{i} \xi_{i}}{\beta+\alpha+\sum_{i=1}^{t} \rho_{i}}
$$

Now, consider the vectors $z$ and $z_{i t}$ where

$$
z=\left[\begin{array}{c}
y \\
\xi_{1} \\
\vdots \\
\xi_{T} \\
\theta
\end{array}\right] \quad z_{i t}=\left[\begin{array}{c}
y \\
\xi_{1} \\
\vdots \\
\xi_{T} \\
x_{i t}
\end{array}\right]
$$


(the difference between $z$ and $z_{i t}$ is that the $\theta$ is replaced by $x_{i t}$ ). Denote by $E_{i t} z$ the conditional expectation of $z$ according to $i$ 's information set at date t. That is,

$$
E_{i t} z=\left[\begin{array}{c}
E_{i t} y \\
E_{i t} \xi_{1} \\
\vdots \\
E_{i t} \xi_{T} \\
E_{i t} \theta
\end{array}\right]
$$

From (11), and since $\xi_{s}$ is either known at date $t$ or is a random variable centred on $\theta$, we can write down a stochastic matrix $B_{t}$ such that

$$
E_{i t} z=B_{t} z_{i t}
$$

The $B_{t}$ matrix will play a crucial role in our analysis. This matrix can be depicted as follows.

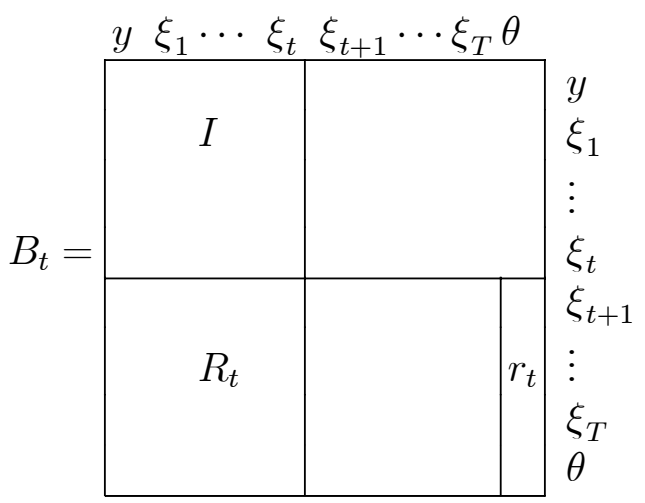

where $I$ is the identity matrix of order $t+1, R_{t}$ is a $(T-t+1) \times(t+1)$ matrix and $r_{t}$ is a $T-t+1$ column vector given by

$$
R_{t}=\left[\begin{array}{cccc}
\alpha_{t} & \rho_{1 t} & \cdots & \rho_{t t} \\
\alpha_{t} & \rho_{1 t} & \cdots & \rho_{t t} \\
\vdots & \vdots & & \vdots \\
\alpha_{t} & \rho_{1 t} & \cdots & \rho_{t t}
\end{array}\right], \quad r_{t}=\left[\begin{array}{c}
\beta_{t} \\
\beta_{t} \\
\vdots \\
\beta_{t}
\end{array}\right]
$$

where

$$
\alpha_{t}=\frac{\alpha}{\alpha+\sum_{i=1}^{t} \rho_{i}+\beta}, \quad \rho_{k t}=\frac{\rho_{k}}{\alpha+\sum_{i=1}^{t} \rho_{i}+\beta}, \quad \beta_{t}=\frac{\beta}{\alpha+\sum_{i=1}^{t} \rho_{i}+\beta}
$$


are respectively the relative precisions of the ex ante mean $y$, the date $k$ price signal at date $t$, and the private signal $x_{i t}$. All other entries in $B_{t}$ are zero. Taking the average over $i$ of both sides of (12),

$$
\bar{E}_{t} z=B_{t} z
$$

since the mean of all the $\left\{x_{i}\right\}$ gives $\theta$. Meanwhile, trader $i$ 's expectation of $\bar{E}_{t} z$ at date $t-1$ is given by

$$
\begin{aligned}
E_{i, t-1} \bar{E}_{t} z & =E_{i, t-1} B_{t} z \\
& =B_{t} E_{i, t-1} z \\
& =B_{t} B_{t-1} z_{i, t-1}
\end{aligned}
$$

Taking averages across $i$ on both sides,

$$
\bar{E}_{t-1} \bar{E}_{t} z=B_{t} B_{t-1} z
$$

Note the reversal of the order of the belief operator when it is converted to the equivalent matrix operation. The intuition for this reversal is the fact that the date $t$ average expectation must be a linear combination of random variables realized up to date $t$. However, since $z$ contains all random variables up to date $T$, the average expectation at date $t$ must put zero weight on any random variable realized later than date $t$. Iterating the relationship (15), we have

$$
\begin{aligned}
\bar{E}_{t} \bar{E}_{t+1} \cdots \bar{E}_{T} z & =B_{T} \cdots B_{t+1} B_{t} z \\
& =B_{t}^{*} z
\end{aligned}
$$

where

$$
B_{t}^{*} \equiv B_{T} \cdots B_{t+1} B_{t}
$$

Since the bottom entry of $z$ is $\theta$, the bottom entry of $B_{t}^{*} z$ thus gives the iterated average expectation of $\theta$. 
The matrix $B_{t}$ can be interpreted as the transition matrix of a Markov chain defined on the set $\left\{y, \xi_{1}, \cdots, \xi_{T}, \theta\right\}$. This suggests that we can use the Markov chain argument with absorbing states used in section 2 to evaluate iterated expectations. However, we can see from (16) that the transition matrix used in each step of the iteration is different. In other words, we have a non-homogenous Markov chain where the transition probabilities change over time. From (13) we can track the transition probabilities. We note the following features of the Markov chain defined by the sequence of transition matrices $B_{T}, B_{T-1}, \cdots, B_{1}$.

- The state $y$ is an absorbing state of this chain. We can see from (13) that once the system has entered this state, it never emerges. Therefore, it gathers more probability mass with each transition.

- The price signals $\xi_{t}$ are "temporary absorbing states" in the sense that initially they act like absorbing states, but after $T-t$ periods in the transition, they suddenly become transient states by giving up all their probability mass to other states

Let us denote by $b_{t}$ the last row of $B_{t}$, and denote by $b_{t}^{*}$ the last row of $B_{t}^{*}$, where

$$
\begin{aligned}
b_{t} & =\left[\alpha_{t}, \rho_{1 t}, \rho_{2 t}, \cdots, \rho_{t t}, 0, \cdots, 0, \beta_{t}\right] \\
b_{t}^{*} & =\left[\alpha_{t}^{*}, \rho_{1 t}^{*}, \rho_{2 t}^{*}, \cdots, \rho_{t t}^{*}, 0, \cdots, 0, \beta_{t}^{*}\right]
\end{aligned}
$$

Let us reiterate the following properties.

Lemma 4 For all $t, E_{s}\left(p_{t}\right)=b_{t}^{*} z$ and $E_{s}\left(\bar{E}_{t}(\theta)\right)=b_{t} z$.

In other words, the mean price path can be calculated using $\left\{B_{t}^{*}\right\}$ while the mean path for $\bar{E}_{t}(\theta)$ can be calculated using $\left\{B_{t}\right\}$. Lemma 4 follow 
directly from (14) and (16). It shows the usefulness of the matrices $B_{t}^{*}$ and $B_{t}$. The aggregate demand for the risky asset at date $t$ is given by

$$
\begin{aligned}
\frac{\tau}{\operatorname{Var}_{t}\left(p_{t+1}\right)}\left(\bar{E}_{t}\left(p_{t+1}\right)-p_{t}\right) & =\frac{\tau}{\operatorname{Var}_{t}\left(p_{t+1}\right)}\left(\bar{E}_{t} \bar{E}_{t+1} \cdots \bar{E}_{T}(\theta)-p_{t}\right) \\
& =\frac{\tau}{\operatorname{Var}_{t}\left(p_{t+1}\right)}\left(b_{t}^{*} z-p_{t}\right)
\end{aligned}
$$

Market clearing then implies that

$$
\begin{aligned}
p_{t} & =b_{t}^{*} z-\frac{\operatorname{Var}_{t}\left(p_{t+1}\right)}{\tau} s_{t} \\
& =\alpha_{t}^{*} y+\sum_{i=1}^{t} \rho_{i t}^{*} \xi_{t}+\beta_{t}^{*} \theta-\frac{\operatorname{Var}_{t}\left(p_{t+1}\right)}{\tau} s_{t}
\end{aligned}
$$

Since $\xi_{t}$ is the sum of $\theta$ and a noise term that depends on the supply noise at date $t$, and since the sum of elements in $b_{t}^{*}$ must sum to 1 , we can write price $p_{t}$ in general as

$$
p_{t}=\alpha_{t}^{*} y+\left(1-\alpha_{t}^{*}\right) \theta-\phi_{t 1} s_{1}-\phi_{t 2} s_{2}-\cdots-\phi_{t t} s_{t}
$$

The following pair of lemmas are proved in appendix A.

Lemma $50<\alpha_{T}^{*}<\cdots<\alpha_{t}^{*}<\alpha_{t-1}^{*}<\cdots<\alpha_{1}^{*}<1$.

Lemma 6 For $t<T$, we have $\alpha_{t}^{*}>\alpha_{t}$. For $T, \alpha_{T}^{*}=\alpha_{T}$.

Then proposition 2 follows from lemma 5 by setting $\lambda_{t}=\alpha_{t}^{*}$. Thus, equilibrium prices exhibit inertia or "drift" in adjusting to the new fundamentals. We can then prove proposition 1, which shows that the mean time path of prices is further away from fundamentals than the mean time path of $\bar{E}_{t}(\theta)$. Using an exactly analogous argument to that used to derive (10), we can write down the time path of average expectations of the fundamentals $\bar{E}_{t}(\theta)$ as

$$
\begin{aligned}
\bar{E}_{1}(\theta)= & \hat{\lambda}_{1} y+\hat{\kappa}_{1} \theta-\hat{\phi}_{11} s_{1} \\
\bar{E}_{2}(\theta)= & \hat{\lambda}_{2} y+\hat{\kappa}_{2} \theta-\hat{\phi}_{21} s_{1}-\hat{\phi}_{22} s_{2} \\
& \vdots \\
\bar{E}_{T}(\theta)= & \hat{\lambda}_{T} y+\hat{\kappa}_{T} \theta-\hat{\phi}_{T 1} s_{1}-\hat{\phi}_{T 2} s_{2}-\cdots-\hat{\phi}_{T T} s_{T}
\end{aligned}
$$


Then lemma 6 shows that $\hat{\lambda}_{t}<\lambda_{t}$ for all $t<T$, but $\hat{\lambda}_{T}=\lambda_{T}$. This proves proposition 1.

The intuition for why $\hat{\lambda}_{t}<\lambda_{t}$ lies in the fact that in the Markov chain defined by the matrices $\left\{B_{t}\right\}$, the state $y$ is an absorbing state. The price $p_{t}$ is determined by the iterated average expectations of $\theta$, and hence in the Markov chain, the probability of being absorbed at $y$ is large, and certainly much larger than the one shot transition implied by the mean path of $\bar{E}_{t}(\theta)$. Thus, the mean price path gives a higher weight to history than does the mean path of $\bar{E}_{t}(\theta)$. This explains both the greater sluggishness of prices, and the systematic deviation of price from average belief of fundamentals.

In appendix B, we present the solution for the two trading period version of our model that uses more direct methods that have much in common with the earlier literature. As well as confirming the general form of the solution identified so far, it also serves to show the respective role of the informational parameters in the solution. In particular, as the precision of the private signal becomes small, or when the noise in the supply becomes large, prices become less precise signals of the fundamental value, and the mean price path follows the naive iterated expectations given by equation (3) in section 2 that ignores the information given by prices. In such cases, the expression in proposition 3 gives us the mean path of price.

\section{Discussion}

Our paper has a number of antecedents that touch upon the main themes that we have introduced here. A number of papers have examined the role of higher order beliefs in asset pricing. However, fully rational models are typically somewhat special and hard to link to standard asset pricing models (see, e.g., Allen, Morris and Postlewaite (1993), Morris, Postlewaite and Shin (1995) and Biais and Boessarts (1998)). Also, our paper is closely related to two recent papers by Bacchetta and Van Wincoop (2002, 2004). The former 
paper presents numerical solutions of asset pricing formulae in the context of foreign exchange markets. The latter paper develops the results of our paper in the direction of deriving a formula for the "higher order wedge" in the asset price - i.e. the difference between the asset price and the average expectation of fundamentals.

A number of authors have noted that agents will not act on private information if they do not expect that private information to be reflected in asset prices at the time that they sell the asset (e.g., Froot, Scharfstein and Stein (1992)). This phenomenon is clearly related to the horizons of traders in the market (see, e.g., Dow and Gorton (1994)). Tirole (1982) emphasized the importance of myopic traders in breaking down the backward induction argument against asset market bubbles. The behavioral approach exploits this feature to the full by assuming that rational (but impatient) traders forecast the beliefs of irrational traders (e.g., De Long, Shleifer, Summers and Waldmann (1990)). But since irrationality is by no means a necessary ingredient for higher order beliefs to matter it seems useful to have a model where rational agents are worried about the forecasts of other rational agents.

The existence of an equivalent martingale measure in the standard representative investor asset pricing model has become a cornerstone of modern finance since the early contributions, such as Harrison and Kreps (1979). In some cases, it is possible to extend the martingale property to differential information economies. For instance, Duffie and Huang (1986) showed that as long as there is one agent who is more informed than any other, we can find an equivalent martingale measure. In general, however, the existence of an equivalent martingale measure cannot be guaranteed. Duffie and Kan (1991) give an example of an economy with true asymmetric information - i.e., no agent who is more informed than any other - where there is no equivalent martingale measure. ${ }^{7}$ In our case, the average expectations operator fails

\footnotetext{
${ }^{7}$ More precisely, there is no "universal equivalent martingale measure" - i.e., no one probability distribution that could be used to price assets conditional on each trader's
} 
to satisfy the law of iterated expectations. So, if the average expectations operator is also the pricing operator, then we know that there cannot be an equivalent martingale measure. This failure of the martingale property is, of course, hardly surprising. What we want to emphasize is that the martingale property fails for average expectations in a systematic way (e.g., there is a bias towards public information). ${ }^{8}$

The arguments that we have presented in this paper combine all the above ingredients. The noisy rational expectations model with short-lived traders exhibits the following features: prices reflect average expectations of average expectations of asset returns; prices are overly sensitive to public information; and traders underweight their private information. Our assumption of overlapping generation of traders is designed to highlight how closely connected these three features are in a standard asset pricing model. We believe they should be linked in a wide array of asset pricing models.

An important issue for future research is the extent to which asset prices in models like the one presented above can be construed as bubbles. Chapter 4 of Shiller (2000) is devoted to the idea that the news media, by propagating information in a public way, may create or exacerbate asset market bubbles by coordinating market participants' expectations. News stories without much information content may play a role akin to "sunspots" - i.e., payoff irrelevant signals that coordinate players' expectations. In our model, if public information suggests that payoffs will be high then this can lead to high asset prices even if many traders have private information that the true value is low. To the extent that market prices are biased signals of the underlying fundamental liquidation value of the asset, our paper may shed light on one important aspect of bubbles in terms of the systematic departure information.

${ }^{8}$ In Harrison and Kreps (1978), the martingale asset pricing formula fails because there are short sales constraints and the asset price depends in each period on the most optimistic expectation in the economy. Most optimistic expectations also fail to satisfy a martingale property in a systematic way (they are a submartingale). 
of prices from the common knowledge value of the asset. In our model, public information exercises a disproportionate influence on the price of the asset - pushing it away from the fundamentals. Having said all this, it is also clear that our model fails to capture many aspects of bubbles as they are conventionally understood, such as the rapid run-up in prices followed by a precipitous crash. Abreu and Brunnermeier (2003) has recently modelled such features using the failure of common knowledge of fundamentals. To the extent that failure of common knowledge of fundamentals is the flip side of higher order uncertainty, Abreu and Brunnermeier's work rests on ideas that are closely related to those explored in our paper.

We conclude with some comments on the short-lived trader assumption. For our analysis, this has been a critical assumption for the methods used. However, even for long-lived traders, if they have a motive for smoothing consumption over time, some of the features of the problem will survive. An alternative way of proceeding would have been to assume that consumption takes place only at the very end of the trading period, and that traders are long-lived. In such contexts, we know from the work of Samuelson (1969) that if traders either have log utility or if they have constant relative risk aversion and the returns are independent, then traders will act as if they are myopic. The relevant question is how much quantitative significance the assumption of short-lived traders has. Simulations in Bacchetta and Van Wincoop (2002) suggest that the difference between the short and long-lived trader assumption may not be very large quantitatively ${ }^{9}$. For these reasons, as well as for the insights gained, our assumption of short-lived traders would be a useful starting point.

\footnotetext{
${ }^{9}$ In an earlier version of our paper (Allen, Morris and Shin (2003)), we explored the differences between the short-lived and long-lived trader model in the limiting case where prices become uninformative. In this limiting case, the first period price in the long-lived model collapses to that in the single period trading model. Thus, in this instance, the difference between the long-lived trader and short-lived trader case is large.
} 


\section{Appendix A}

Proofs of lemmas 5 and 6 . We can verify from the product $B_{t}^{*} B_{t-1}$ that

$$
\begin{aligned}
\alpha_{t-1}^{*} & =\alpha_{t}^{*}+\alpha_{t-1}\left(\rho_{t t}^{*}+\beta_{t}^{*}\right) \\
\rho_{s, t-1}^{*} & =\rho_{s, t}^{*}+\rho_{s, t-1}\left(\rho_{t t}^{*}+\beta_{t}^{*}\right), \quad \text { for } s \leq t-1 \\
\beta_{t-1}^{*} & =\beta_{t-1}\left(\rho_{t t}^{*}+\beta_{t}^{*}\right)
\end{aligned}
$$

This defines a dynamic system whose boundary conditions are given by

$$
\begin{aligned}
& \alpha_{T}^{*}=\alpha_{T} \\
& \rho_{s T}^{*}=\rho_{s T}, \quad \text { for } s \leq T \\
& \beta_{T}^{*}=\beta_{T}
\end{aligned}
$$

We can see that $\alpha_{t}^{*}$ is a decreasing function of $t$. This proves lemma 5. To prove lemma 6 , let us first prove that $\rho_{s t}^{*}>\rho_{s t}$ for $s \leq t<T$. The proof is by induction backwards from $T$. From (19) and (20),

$$
\begin{aligned}
\rho_{s, T-1}^{*} & =\rho_{s, T}^{*}+\rho_{s, T-1}\left(\rho_{T T}^{*}+\beta_{T}^{*}\right) \\
& =\rho_{s, T}+\rho_{s, T-1}\left(\rho_{T T}+\beta_{T}\right) \\
& =\rho_{s, T-1}\left(1+\beta_{T}\right) \\
& >\rho_{s, T-1}
\end{aligned}
$$

For the inductive step, suppose $\rho_{s t}^{*}>\rho_{s t}$. Then

$$
\begin{aligned}
\rho_{s, t-1}^{*} & =\rho_{s, t}^{*}+\rho_{s, t-1}\left(\rho_{t t}^{*}+\beta_{t}^{*}\right) \\
& >\rho_{s, t}+\rho_{s, t-1}\left(\rho_{t t}+\beta_{t}^{*}\right) \\
& =\rho_{s, t-1}\left(1+\beta_{t}^{*}\right) \\
& >\rho_{s, t-1}
\end{aligned}
$$

This proves that $\rho_{s t}^{*}>\rho_{s t}$ for $s \leq t<T$. From $(20) \alpha_{T}^{*}=\alpha_{T}$. Then,

$$
\begin{aligned}
\alpha_{T-1}^{*} & =\alpha_{T}^{*}+\alpha_{T-1}\left(\rho_{T T}^{*}+\beta_{T}^{*}\right) \\
& =\alpha_{T}+\alpha_{T-1}\left(\rho_{T T}+\beta_{T}\right) \\
& =\alpha_{T-1}\left(1+\beta_{T}\right)>\alpha_{T-1}
\end{aligned}
$$


For the inductive step, suppose that $\alpha_{t}^{*}>\alpha_{t}$. Then

$$
\begin{aligned}
a_{t-1}^{*} & =\alpha_{t}^{*}+\alpha_{t-1}\left(\rho_{t t}^{*}+\beta_{t}^{*}\right) \\
& >\alpha_{t}+\alpha_{t-1}\left(\rho_{t t}+\beta_{t}^{*}\right) \\
& =\alpha_{t-1}\left(1+\beta_{t}^{*}\right)>\alpha_{t-1}
\end{aligned}
$$

where the second inequality follows from the fact that $\rho_{t t}^{*}>\rho_{t t}$. This proves lemma 6.

Proof of Proposition 3. We begin by demonstrating a result that has some independent interest. We will show that the information value of price $p_{t}$ depends in a simple way on risk tolerance $\tau$ and the weight given to the private signal $x_{i t}$ in forming demands. Suppose price take the form

$$
p_{t}=\pi_{t} E\left(\theta \mid y, \xi_{1}, \cdots, \xi_{t-1}\right)+\mu_{t} \theta-\psi_{t} s_{t}
$$

The aggregate demand at date $t$ is given by

$$
\begin{aligned}
& \frac{\tau}{\operatorname{Var}_{t}\left(p_{t+1}\right)}\left(b_{t}^{*} z-p_{t}\right) \\
= & \frac{\tau}{\operatorname{Var}_{t}\left(p_{t+1}\right)}\left(\sum_{i=i}^{t-1} \rho_{i} \xi_{i}+\rho_{t} \xi_{t}+\beta_{t} \theta-p_{t}\right)
\end{aligned}
$$

Setting this equal to supply $s_{t}$, solving for $p$ and comparing coefficients with (21), we have $\mu_{t}=\rho_{t}+\beta_{t}$ and $\psi_{t}=\frac{\rho_{t}+\beta_{t}}{\tau \beta_{t}} \operatorname{Var}_{t}\left(p_{t+1}\right)$. Thus the random variable

$$
\frac{p_{t}-\pi_{t} E\left(\theta \mid y, \xi_{1}, \cdots, \xi_{t-1}\right)}{\mu}=\theta-\frac{\psi_{t}}{\mu_{t}} s_{t}
$$

has variance

$$
\frac{1}{\gamma}\left(\frac{\operatorname{Var}_{i t}\left(p_{t+1}\right)}{\tau \beta_{t}^{*}}\right)^{2}
$$

which goes to infinity as $\tau \rightarrow 0$. In this limit, prices lose all their information value. The precisions $\left\{\rho_{t}\right\}$ all tend to zero, and the dynamical system above reduces to the pair

$$
\begin{aligned}
\alpha_{t-1}^{*} & =\alpha_{t}^{*}+\alpha_{t-1} \beta_{t}^{*} \\
\beta_{t}^{*} & =\beta_{t} \beta_{t+1}^{*}
\end{aligned}
$$


where $\beta_{t}=\beta /(\alpha+\beta)$ for any $t$. Thus, $\beta_{t}^{*}=(\beta /(\alpha+\beta))^{T-t+1}$. We claim that

$$
\alpha_{t}^{*}=1-\left(\frac{\beta}{\alpha+\beta}\right)^{T-t+1}
$$

The proof is by induction backwards from $T$. For $T$, we have $\alpha_{T}^{*}=\alpha_{T}=$ $\alpha /(\alpha+\beta)=1-(\beta /(\alpha+\beta))$. For $t$,

$$
\begin{aligned}
\alpha_{t}^{*} & =\alpha_{t+1}^{*}+\frac{\alpha}{\alpha+\beta}\left(\frac{\beta}{\alpha+\beta}\right)^{T-t} \\
& =1-\left(\frac{\beta}{\alpha+\beta}\right)^{T-t}+\frac{\alpha}{\alpha+\beta}\left(\frac{\beta}{\alpha+\beta}\right)^{T-t} \\
& =1-\left(\frac{\beta}{\alpha+\beta}\right)^{T-t+1}
\end{aligned}
$$

This proves proposition 3 . 


\section{ApPendix B}

In this appendix, we solve our model for the two period trading case. The solution is in four steps.

STEP 1: LEARNING FROM FIRST PERIOD PRICES

Assume that period 1 prices follow a linear rule

$$
p_{1}=\kappa_{1}\left(\lambda_{1} y+\mu_{1} \theta-s_{1}\right)
$$

Observe that

$$
\frac{1}{\kappa_{1} \mu_{1}}\left(p_{1}-\kappa_{1} \lambda_{1} y\right)=\theta-\frac{1}{\mu_{1}} s_{1}
$$

So

$$
\frac{1}{\kappa_{1} \mu_{1}}\left(p_{1}-\kappa_{1} \lambda_{1} y\right)
$$

is distributed normally with mean $\theta$ and precision $\mu_{1}^{2} \gamma_{1}$. Thus at period 1 , based on prior information alone, $\theta$ is distributed normally with mean

$$
\begin{aligned}
\bar{y}_{2} & =\frac{\alpha y+\frac{\mu_{1} \gamma_{1}}{\kappa_{1}}\left(p_{1}-\kappa_{1} \lambda_{1} y\right)}{\alpha+\mu_{1}^{2} \gamma_{1}} \\
& =\frac{\left(\alpha-\mu_{1} \gamma_{1} \lambda_{1}\right) y+\frac{\mu_{1} \gamma_{1}}{\kappa_{1}} p_{1}}{\alpha+\mu_{1}^{2} \gamma_{1}}
\end{aligned}
$$

and precision

$$
\bar{\alpha}_{2}=\alpha+\mu_{1}^{2} \gamma_{1}
$$

Trader $i$ who in addition observes private signal $x_{i}$ will believe that $\theta$ is normally distributed with mean

$$
E_{i}^{1}(\theta)=\frac{\left(\alpha-\mu_{1} \gamma_{1} \lambda_{1}\right) y+\beta x_{i}+\frac{\mu_{1} \gamma_{1}}{\kappa_{1}} p_{1}}{\alpha+\beta+\mu_{1}^{2} \gamma_{1}}
$$

and precision

$$
\alpha+\beta+\mu_{1}^{2} \gamma_{1}
$$

STEP 2: LEARNING FROM SECOND PERIOD PRICES

Now assume that second period prices follow a linear rule:

$$
p_{2}=\kappa_{2}\left(\lambda_{2} \bar{y}_{2}+\mu_{2} \theta-s_{2}\right)
$$


Hence

$$
\frac{1}{\kappa_{2} \mu_{2}}\left(p_{2}-\kappa_{2} \lambda_{2} \bar{y}_{2}\right)
$$

is distributed normally with mean $\theta$ and precision $\mu_{2}^{2} \gamma_{2}$.

Trader $i$ who in addition observes private signal $x_{i}$ will believe that $\theta$ is normally distributed with mean

$$
\begin{aligned}
& E_{i}^{2}(\theta)= \\
& \left(\frac{\bar{\alpha}_{2}-\lambda_{2} \mu_{2} \gamma_{2}}{\bar{\alpha}_{2}+\beta+\mu_{2}^{2} \gamma_{2}}\right) \bar{y}_{2}+\left(\frac{\beta}{\bar{\alpha}_{2}+\beta+\mu_{2}^{2} \gamma_{2}}\right) x_{i}+\left(\frac{\frac{\mu_{2} \gamma_{2}}{\kappa_{2}}}{\bar{\alpha}_{2}+\beta+\mu_{2}^{2} \gamma_{2}}\right) p_{2} \\
& =\left\{\begin{array}{c}
\left(\frac{\alpha+\mu_{1}^{2} \gamma_{1}-\lambda_{2} \mu_{2} \gamma_{2}}{\alpha+\mu_{1}^{2} \gamma_{1}+\beta+\mu_{2}^{2} \gamma_{2}}\right)\left(\frac{\left(\alpha-\mu_{1} \gamma_{1} \lambda_{1}\right) y+\frac{\mu_{1} \gamma_{1}}{\kappa_{1}} p_{1}}{\alpha+\mu_{1}^{2} \gamma_{1}}\right) \\
+\left(\frac{\beta}{\alpha+\mu_{1}^{2} \gamma_{1}+\beta+\mu_{2}^{2} \gamma_{2}}\right) x_{i} \\
+\left(\frac{\frac{\mu_{2} \gamma_{2}}{\kappa_{2}}}{\alpha+\mu_{1}^{2} \gamma_{1}+\beta+\mu_{2}^{2} \gamma_{2}}\right) p_{2}
\end{array}\right\} \\
& =\left\{\begin{array}{c}
\left(\frac{\alpha+\mu_{1}^{2} \gamma_{1}-\lambda_{2} \mu_{2} \gamma_{2}}{\alpha+\mu_{1}^{2} \gamma_{1}+\beta+\mu_{2}^{2} \gamma_{2}}\right)\left(\frac{\alpha-\mu_{1} \gamma_{1} \lambda_{1}}{\alpha+\mu_{1}^{2} \gamma_{1}}\right) y \\
\left(\frac{\alpha+\mu_{1}^{2} \gamma_{1}-\lambda_{2} \mu_{2} \gamma_{2}}{\alpha+\mu_{1}^{2} \gamma_{1}+\beta+\mu_{2}^{2} \gamma_{2}}\right) \frac{\mu_{1} \gamma_{1}}{\kappa_{1}\left(\alpha+\mu_{1}^{2} \gamma_{1}\right)} p_{1} \\
+\left(\frac{\beta}{\alpha+\mu_{1}^{2} \gamma_{1}+\beta+\mu_{2}^{2} \gamma_{2}}\right) x_{i} \\
+\left(\frac{\frac{\mu_{2} \gamma_{2}}{\alpha+\mu_{1}^{2} \gamma_{1}+\beta+\mu_{2}^{2} \gamma_{2}}}{\alpha+\mu_{1}}\right) p_{2}
\end{array}\right\}
\end{aligned}
$$

and precision

$$
\begin{gathered}
\bar{\alpha}_{2}+\beta+\mu_{2}^{2} \gamma_{2} \\
=\alpha+\mu_{1}^{2} \gamma_{1}+\beta+\mu_{2}^{2} \gamma_{2} .
\end{gathered}
$$

STEP 3: SOLVING FOR SECOND PERIOD PRICES

Trader $i$ 's demand for the asset will be $\tau\left(\bar{\alpha}_{2}+\beta+\mu_{2}^{2} \gamma_{2}\right)\left[\left(\frac{\bar{\alpha}_{2}-\lambda_{2} \mu_{2} \gamma_{2}}{\bar{\alpha}_{2}+\beta+\mu_{2}^{2} \gamma_{2}}\right) \bar{y}_{2}+\left(\frac{\beta}{\bar{\alpha}_{2}+\beta+\mu_{2}^{2} \gamma_{2}}\right) x_{i}+\left(\frac{\frac{\mu_{2} \gamma_{2}}{\kappa_{2}}}{\bar{\alpha}_{2}+\beta+\mu_{2}^{2} \gamma_{2}}\right) p_{2}-p_{2}\right]$

Collecting terms and simplifying, we have

$$
\tau\left[\left(\bar{\alpha}_{2}-\lambda_{2} \mu_{2} \gamma_{2}\right) \bar{y}_{2}+\beta x_{i}-\left(\bar{\alpha}_{2}+\beta+\mu_{2} \gamma_{2}\left(\mu_{2}-\frac{1}{\kappa_{2}}\right)\right) p_{2}\right]
$$


Total demand for the asset will be

$$
\tau\left[\left(\bar{\alpha}_{2}-\lambda_{2} \mu_{2} \gamma_{2}\right) \bar{y}_{2}+\beta \theta-\left(\bar{\alpha}_{2}+\beta+\mu_{2} \gamma_{2}\left(\mu_{2}-\frac{1}{\kappa_{2}}\right)\right) p_{2}\right]
$$

Market clearing implies that this equals $s_{2}$, i.e., rearranging,

$$
p_{2}=\frac{\left(\bar{\alpha}_{2}-\lambda_{2} \mu_{2} \gamma_{2}\right) \bar{y}_{2}+\beta \theta-\frac{1}{\tau} s_{2}}{\bar{\alpha}_{2}+\beta+\mu_{2} \gamma_{2}\left(\mu_{2}-\frac{1}{\kappa_{2}}\right)}
$$

So

$$
\begin{aligned}
\mu_{2} & =\tau \beta \\
\lambda_{2} & =\frac{\bar{\alpha}_{2} \tau}{1+\beta \gamma_{2} \tau^{2}}=\frac{\left(\alpha+\mu_{1}^{2} \gamma_{1}\right) \tau}{1+\beta \gamma_{2} \tau^{2}} \\
\kappa_{2} & =\frac{\frac{1}{\tau}+\tau \beta \gamma_{2}}{\bar{\alpha}_{2}+\beta+\tau^{2} \beta^{2} \gamma_{2}}=\frac{\frac{1}{\tau}+\tau \beta \gamma_{2}}{\alpha+\mu_{1}^{2} \gamma_{1}+\beta+\tau^{2} \beta^{2} \gamma_{2}}
\end{aligned}
$$

This is implies that the second period price is normally distributed with mean

$$
\frac{\alpha+\mu_{1}^{2} \gamma_{1}}{\alpha+\mu_{1}^{2} \gamma_{1}+\beta+\tau^{2} \beta^{2} \gamma_{2}} y+\frac{\beta\left(1+\tau^{2} \beta \gamma_{2}\right)}{\alpha+\mu_{1}^{2} \gamma_{1}+\beta+\tau^{2} \beta^{2} \gamma_{2}} \theta
$$

and variance

$$
\left(\frac{\frac{1}{\tau}+\tau \beta \gamma_{2}}{\alpha+\mu_{1}^{2} \gamma_{1}+\beta+\tau^{2} \beta^{2} \gamma_{2}}\right)^{2} \frac{1}{\gamma_{2}} .
$$

Define $z$ as

$$
z \equiv \frac{\beta\left(1+\tau^{2} \beta \gamma_{2}\right)}{\alpha+\mu_{1}^{2} \gamma_{1}+\beta+\tau^{2} \beta^{2} \gamma_{2}}
$$

Then, $p_{2}$ can be written as a linear combination of $y, \theta$ and $s_{2}$ where

$$
p_{2}=(1-z) y+z \theta-\frac{s_{2}}{\tau\left(\bar{\alpha}_{2}+\beta+\mu_{2} \gamma_{2}\left(\mu_{2}-\frac{1}{\kappa_{2}}\right)\right)}
$$

Integrating out the supply shock $s_{2}$, we have that the mean of $p_{2}$ is

$$
(1-z) y+z \theta
$$

STEP 4: SOLVING FOR FIRST PERIOD PRICES

For the short-lived trader, the demand for the asset in period 1 is

$$
\frac{\tau\left(E_{1 i}\left(p_{2}\right)-p_{1}\right)}{\operatorname{Var}_{1 i}\left(p_{2}\right)}
$$


where $E_{1 i}\left(p_{2}\right)$ is the $i$ 's conditional expectation of $p_{2}$ at date 1 and $\operatorname{Var}_{1 i}\left(p_{2}\right)$ is $i$ 's conditional variance of $p_{2}$ at date 1 . From (28), trader $i$ 's demand is given by

$$
\begin{aligned}
& \frac{\tau\left(z E_{1 i}(\theta)+(1-z) y-p_{1}\right)}{\operatorname{Var}_{1 i}\left(p_{2}\right)} \\
= & \frac{\tau}{\operatorname{Var}_{1 i}\left(p_{2}\right)}\left(z\left(\frac{\left(\alpha-\mu_{1} \lambda_{1} \gamma_{1}\right) y+\beta x_{i}+\frac{\mu_{1} \gamma_{1}}{\kappa_{1}} p_{1}}{1 / \operatorname{Var}_{i 1}(\theta)}\right)+(1-z) y-p_{1}\right)
\end{aligned}
$$

where

$$
\operatorname{Var}_{1 i}(\theta)=\frac{1}{\alpha+\beta+\mu_{1}^{2} \gamma_{1}}
$$

is $i$ 's conditional variance of $\theta$ based on information at date 1 . The conditional variances $\operatorname{Var}_{1 i}\left(p_{2}\right)$ and $\operatorname{Var}_{1 i}(\theta)$ are identical across traders, and so we can write them simply as $\operatorname{Var}_{1}\left(p_{2}\right)$ and $\operatorname{Var}_{1}(\theta)$. Integrating over all traders, the aggregate demand is given by

$$
\frac{\tau \operatorname{Var}_{1}(\theta)}{\operatorname{Var}_{1}\left(p_{2}\right)}\left\{\begin{array}{l}
y\left[z\left(\alpha-\mu_{1} \lambda_{1} \gamma_{1}\right)+(1-z)\left(\alpha+\beta+\mu_{1}^{2} \gamma_{1}\right)\right] \\
+z \beta \theta \\
-p_{1}\left[\left(\alpha+\beta+\mu_{1}^{2} \gamma_{1}\right)-z \frac{\mu_{1} \gamma_{1}}{\kappa_{1}}\right]
\end{array}\right\}
$$

Market clearing implies that this is equal to $s_{1}$. Rearranging in terms of $p_{1}$ and comparing coefficients with (22) we have

$$
\begin{aligned}
\kappa_{1} \mu_{1} & =z\left(\frac{\beta+\mu_{1}^{2} \gamma_{1}}{\alpha+\beta+\mu_{1}^{2} \gamma_{1}}\right) \\
\kappa_{1} \lambda_{1} & =1-z\left(\frac{\beta+\mu_{1}^{2} \gamma_{1}}{\alpha+\beta+\mu_{1}^{2} \gamma_{1}}\right) \\
\kappa_{1} & =\frac{\operatorname{Var}_{1}\left(p_{2}\right)}{\tau \operatorname{Var}_{1}(\theta)\left[\left(\alpha+\beta+\mu_{1}^{2} \gamma_{1}\right)-z \frac{\mu_{1} \gamma_{1}}{\kappa_{1}}\right]}
\end{aligned}
$$

Thus, defining

$$
w \equiv \frac{\beta+\mu_{1}^{2} \gamma_{1}}{\alpha+\beta+\mu_{1}^{2} \gamma_{1}}
$$

we can express first period price as a linear combination of $y, \theta$ and $s_{1}$ where

$$
p_{1}=(1-w z) y+w z \theta-s_{1} \frac{\operatorname{Var}_{1}\left(p_{2}\right)}{\tau \operatorname{Var}_{1}(\theta)\left[\left(\alpha+\beta+\mu_{1}^{2} \gamma_{1}\right)-z \frac{\mu_{1} \gamma_{1}}{\kappa_{1}}\right]}
$$

Integrating out the supply noise $s_{1}$, we have

$$
E_{s}\left(p_{1}\right)=(1-w z) y+w z \theta
$$




\section{References}

[1] Abreu, D. and M. Brunnermeier (2003) "Bubbles and Crashes" Econometrica, 71, 173-204.

[2] Admati, A. R. (1985) "A Noisy Rational Expectations Equilibrium for Multi-Asset Securities Markets" Econometrica, 53, 629-657.

[3] Allen, F. and G. Gorton (1993). "Churning Bubbles," Review of Economic Studies 60, 813-836.

[4] Allen, F., S. Morris and A. Postlewaite (1993). "Finite Bubbles with Short Sales Constraints and Asymmetric Information," Journal of Economic Theory 61, 206-229.

[5] Allen, F., S. Morris and H. S. Shin (2003) "Beauty Contests, Bubbles and Iterated Expectations in Asset Markets" SSRN discussion paper, http://papers.ssrn.com/sol3/papers.cfm?abstract_id=386083

[6] Amato, J. and H. S. Shin (2003). "Imperfect Common Knowledge and the Information Value of Prices" unpublished paper, LSE.

[7] Ausubel, L. (1990). "Partially-Revealing Rational Expectations Equilibrium in a Competitive Economy," Journal of Economic Theory 50, 93-126.

[8] Bacchetta, P. and E. van Wincoop (2002). "Can Information Heterogeneity Explain the Exchange Rate Determination Puzzle?" unpublished paper, Studienzentrum Gerzensee.

[9] Bacchetta, P. and E. van Wincoop (2004) "Higher Order Expectations in Asset Pricing" unpublished paper, Studienzentrum Gerzensee.

[10] Biais, B. and P. Bossaerts (1998). "Asset Prices and Trading Volume in a Beauty Contest," Review of Economic Studies 65, 307-340. 
[11] Brown, D. and R. Jennings (1989). "On Technical Analysis," Review of Financial Studies 2, 527-551.

[12] Brunnermeier, M. (2001). Asset Pricing Under Asymmetric Information. Oxford: Oxford University Press.

[13] De Long, B., A. Shleifer, L. Summers and R. Waldmann (1990). "Positive Feedback Investment Strategies and Destabilizing Rational Speculation," Journal of Finance 45, 379-395.

[14] Diamond, D. and R. Verrecchia (1981). "Information Aggregation in a Noisy Rational Expectations Economy," Journal of Financial Economics 9, 221-235.

[15] Dow, J. and G. Gorton (1994). "Arbitrage Chains," Journal of Finance 49, 819-849.

[16] Duffie, D. and C.-F. Huang (1986). "Multiperiod Security Markets with Differential Information," Journal of Mathematical Economics 15, 283303.

[17] Duffie, D. and R. Kan (1991). "Universal State Prices and Asymmetric Information."

[18] Froot, K., D. Scharfstein and J. Stein (1992). "Herd on the Street: Informational Inefficiencies in a Market with Short-Term Speculation," Journal of Finance 47, 1461-1484.

[19] Frydman, R. and E. Phelps (1986). Individual Forecasting and Aggregate Outcomes: "Rational Expectations" Examined. Cambridge: Cambridge University Press.

[20] Grossman, S. (1976). "On the Efficiency of Competitive Stock Markets where Traders Have Diverse Information," Journal of Finance 31, 573585. 
[21] Grundy, B. and M. McNichols (1989). "Trade and Revelation of Information through Prices and Direct Disclosure," Review of Financial Studies 2, 495-526.

[22] Harrison M. and D. Kreps (1978). "Speculative Investor Behavior in a Stock Market with Heterogeneous Expectations," Quarterly Journal of Economics XCII, 323-336.

[23] Harrison M. and D. Kreps (1979). "Martingales and Arbitrage in Multiperiod Securities Markets," Journal of Economic Theory 2, 381-408.

[24] He, H. and J. Wang (1995). "Differential Information and Dynamic Behavior of Stock Trading Volume," Review of Financial Studies 8, 914972.

[25] Hellwig, C. (2002). "Public Announcements, Adjustment Delays and the Business Cycle." unpublished paper, UCLA.

[26] Hellwig, M. (1980). "On the Aggregation of Information in Competitive Markets," Journal of Economic Theory 22, 477-498.

[27] Keynes, J. M. (1936). The General Theory of Employment, Interest and Money. London: Macmillan.

[28] Lucas, Robert E. (1972) "Expectations and the Neutrality of Money" Journal of Economic Theory, 4, 103-24.

[29] Lucas, Robert E. (1973) "Some International Evidence on OutputInflation Tradeoffs" American Economic Review, 63, 326-34.

[30] Morris, S. and H. S. Shin (2002). "The Social Value of Public Information," American Economic Review, 92, 1521-1534.

[31] Morris, S., H. S. Shin and A. Postlewaite (1995). "Depth of Knowledge and the Effect of Higher Order Uncertainty," Economic Theory 6, 453467. 
[32] Pearlman, J. and T. Sargent (2002). "Knowing the Forecasts of Others." unpublished paper, NYU.

[33] Phelps, E. (1970) "Introduction" in E. S. Phelps et al. (eds) Microeconomic Foundations of Employment and Information Theory, pp 1- 23, Norton, New York.

[34] Phelps, E. (1983) "The Trouble with "Rational Expectations" and the Problem of Inflation Stabilization" in R. Frydman and E. S. Phelps (eds) Individual Forecasting and Aggregate Outcomes, Cambridge University Press, New York.

[35] Samet, D. (1998). "Iterated Expectations and Common Priors," Games and Economic Behavior 24, 131-141.

[36] Samuelson P. (1969) "Lifetime Portfolio Selection by Dynamic Stochastic Programming" Review of Economics and Statistics, 51, 239-246.

[37] Sargent, T. J. (1991) "Equilibrium with Signal Extraction from Endogenous Variables" Journal of Economic Dynamics and Control, 15, 245-273.

[38] Shiller, R. (2000). Irrational Exuberance. Princeton: Princeton University Press.

[39] Singleton, K. (1987). "Asset Prices in a Time-Series Model with Disparately Informed, Competitive Traders," in New Approaches to Monetary Economics, Proceedings of the Second International Symposium in Economic Theory and Econometrics eds. W. Barnet and K. Singleton, Cambridge University Press, Cambridge, England.

[40] Stasavage, D. (2002). "Communication, Coordination and Common Knowledge in Monetary Policy: Implications for the Euro Zone," unpublished paper, LSE. 
[41] Tirole, J. (1982). "On the Possibility of Speculation Under Rational Expectations," Econometrica 50, 1163-1181.

[42] Townsend, R. (1978) "Market Anticipations, Rational Expectations and Bayesian Analysis" International Economic Review, 19, 481-94.

[43] Townsend, R. (1983). "Forecasting the Forecasts of Others," Journal of Political Economy 91, 546-588.

[44] Wang, J. (1993) "A Model of Intertemporal Asset Prices Under Asymmetric Information" Review of Economic Studies, 60, 249-282.

[45] Wang, J. (1994). "A Model of Competitive Stock Trading Volume," Journal of Political Economy 102, 127-168.

[46] Woodford, M. (2003). "Imperfect Common Knowledge and the Effects of Monetary Policy." in Knowledge, Information and Expectations in Modern Macroeconomics: In Honor of Edmund S. Phelps, P. Aghion, R.Frydman, J. Stiglitz and M. Woodford (eds), Princeton University Press. 